\title{
The impact of the time of drug administration on the effectiveness of combined treatment of hypercholesterolemia with Rosuvastatin and Ezetimibe (RosEze): study protocol for a randomized controlled trial
}

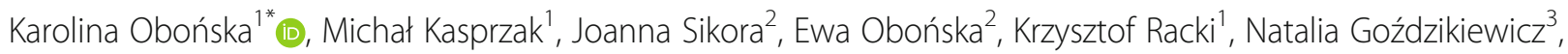
Magdalena Krintus ${ }^{4}$ and Jacek Kubica ${ }^{1}$

\begin{abstract}
Background: Hypercholesterolemia is one of the main risk factors for cardiovascular disease. The first line treatment for hypercholesterolemia is statin therapy. When the expected low-density lipoprotein cholesterol (LDL-C) concentration is not achieved, the pharmacotherapy may be extended by combining the statin with the cholesterol absorption inhibitor ezetimibe.

Methods/design: The study is designed as a randomized, open-label, single-center, crossover study evaluating the effectiveness of combined therapy with rosuvastatin and ezetimibe for hypercholesterolemia. The study is planned to include 200 patients with hypercholesterolemia ineffectively treated with statins for at least 6 weeks. After enrollment participants are randomized into one of two arms receiving rosuvastatin and ezetimibe. In the first arm the study drug is administered in the morning $(8: 00 \mathrm{am})$ for 6 weeks and then in the evening for the next 6 weeks; in the second arm the study drug is administered at first in the evening $(8: 00 \mathrm{pm})$ for the first 6 weeks and then in the morning for the following 6 weeks. In order to minimize non-adherence to the treatment, all patients will receive the study drug free of charge. The primary outcome of the study is change in LDL-C at 6 and 12 weeks of the treatment, depending on the time of day of study drug administration. The secondary endpoints include change in total cholesterol, high-density lipoprotein (HDL) cholesterol, triglycerides, apolipoproteins ApoB and Apo Al, non-HDL cholesterol, small, dense (sd)-LDL cholesterol, lipoprotein(a), glucose, glycated hemoglobin, highsensitivity C-reactive protein, aspartate aminotransferase, alanine aminotransferase, gamma-glutamyl transferase, and creatine kinase at 6 and 12 weeks of the study drug treatment, as well as assessment of plasma fluorescence using stationary and time-resolved fluorescence spectroscopy at baseline and at 6 and 12 weeks of the therapy. (Continued on next page)
\end{abstract}

\footnotetext{
* Correspondence: kalaobonska@op.pl

${ }^{1}$ Department of Cardiology and Internal Medicine, Nicolaus Copernicus

University, Collegium Medicum, 9 Skłodowskiej-Curie Street, 85-094

Bydgoszcz, Poland

Full list of author information is available at the end of the article
} 
(Continued from previous page)

Discussion: The RosEze trial is expected to demonstrate whether there is a significant difference in the effectiveness of the lipid-lowering therapy in reducing the concentration of cholesterol when the medications are taken in the morning compared with the evening time of day.

Trial registration: ClinicalTrials.gov, NCT02772640. Registered on 28 March 2016.

Keywords: Adherence, Cardiovascular risk, Coronary artery disease, Dyslipidemia, Ezetimibe, HydroxymethylglutarylCoA, Hypercholesterolemia, Morning/evening dosing, LDL-C, Rosuvastatin

\section{Background}

Hypercholesterolemia is one of the main risk factors for cardiovascular disease (CVD) [1]. Despite enormous progress in the treatment of coronary artery disease (CAD), patients after surviving their first episode are at risk of recurrence [2]. Hypercholesterolemia is a modifiable risk factor for CVD. Lifestyle changes [3], increased daily physical activity [4-7], as well as optimized diet [8-12] may lead to normalization of specific cholesterol fractions. This strategy, however, often fails or is not sufficient, thus providing the need for pharmacotherapy. The current guidelines recommend statins as the first choice drugs for the treatment of hypercholesterolemia up to the highest recommended dose or the highest tolerable dose (class of recommendation I, level of evidence A) [2]. According to a meta-analysis of studies assessing statins, each $1.0 \mathrm{mmol} / \mathrm{L}(\sim 40 \mathrm{mg} / \mathrm{dL})$ reduction in low-density lipoprotein cholesterol (LDL-C) corresponds to a $10 \%$ reduction in all-cause mortality and a $20 \%$ reduction in the number of deaths from CAD [13]. Furthermore, each $1 \mathrm{mmol} / \mathrm{L}(40 \mathrm{mg} / \mathrm{dL})$ reduction in LDL-C translates into a $23 \%$ and $17 \%$ reduction of the risk of major coronary events and stroke, respectively. Similar results concerning the efficacy and safety of lipid-lowering therapy using statins were obtained in meta-analyses of studies on primary prevention [14-16]. Statins are a heterogeneous group of drugs with respect to their LDL-C reduction power. So far, the most potent statin is rosuvastatin. However, despite intensive statin therapy, only a small group of patients (approximately 20\%) reach the therapeutic lipid-lowering goal [17-20]. When the LDL-C goal is not achieved, the combination of statin with a cholesterol absorption inhibitor - ezetimibe - may be considered (class of recommendation IIa, level of evidence B) [2]. Statin dose titration seems to be less effective compared with the combined therapy with statin and ezetimibe [21]. The combination of statin with ezetimibe reduces the LDL-C by an additional $15-20 \%$ [22].

Unfortunately, despite a wealth of evidence on the efficacy and effectiveness of statins in both primary and secondary prevention, statin adherence remains a consistent barrier, with rates below 50\% demonstrated in several studies [2, 23]. Adherence declines over the duration of treatment [2, 24-28], and this phenomenon is even more pronounced in patients treated for primary compared with secondary prevention of CVD [2]. It was demonstrated in a systematic review and meta-analysis that poor adherence is not limited to statins but to all medications used in secondary prevention for CVD [2, 29, 30]. Furthermore, non-adherence translates into increased healthcare costs of morbidity, hospital readmissions, and mortality [31-35]. There are many determinants of non-adherence to different medications including statins [36-38]. One of the reasons for non-adherence is a large number of drugs taken daily by the patient. Thus, more benefit is achieved with combined drugs containing statin and ezetimibe. Tablets comprising both of these drugs (statin and ezetimibe) simplify the drug administration and increase the probability of drug compliance. Furthermore, the use of these tablets may translate into increased probability for achieving therapeutic goals in hypercholesterolemia treatment [39]. Taking into account the metabolism of cholesterol and possible drug-drug interactions, it is recommended to administer simvastatin in the evening [40]. Rosuvastatin can be administered at any time of the day [41]. In our everyday practice we meet many patients with hypercholesterolemia treated with statins. All of them take the statin in the evening; whereas, for combined treatment with ezetimibe, they take the latter in the morning.

Until now there were no studies assessing the effectiveness of the combined treatment of hypercholesterolemia with rosuvastatin and ezetimibe according to the timing of the drug administration. To fill this evidence gap, the goals of this study are to determine whether the time of the day of rosuvastatin and ezetimibe administration plays any role in the effectiveness of the drug, and to identify side effects of combined therapy with rosuvastatin and ezetimibe administered at the same time of the day. Furthermore, we aim to assess whether administration of lipid-lowering drugs in the morning improves adherence compared with their evening administration.

Considering the potency of rosuvastatin, which is further enhanced when administered in combination with ezetimibe, we expect significant reduction of LDL-C concentration. However, the role of the time of drug administration in this case is questionable and worth further evaluation. 


\section{Methods}

The study is designed as a randomized, open-label, single-center, crossover study evaluating the effectiveness of combined therapy with rosuvastatin and ezetimibe for hypercholesterolemia. The study is conducted with full respect to regulations established in the Declaration of Helsinki. The eligibility criteria for enrollment into the study include adult patients with hypercholesterolemia defined according to the European guidelines [2] and ineffectiveness of statin monotherapy in the treatment of hypercholesterolemia after at least 6 weeks. All study participants will have been on statin therapy due to secondary prevention indications. Furthermore, they are eligible for the study when, despite statin monotherapy, the LDL-C concentration is higher than $70 \mathrm{mg} / \mathrm{dL}$. Key exclusion criteria include the following: active liver disease; unexplained persistent increase in serum transaminase levels, including more than three times the upper limit of normal activity of one of them; severe renal impairment (creatinine clearance $<30 \mathrm{~mL} / \mathrm{min}$ ); myopathy; concomitant treatment with cyclosporine and gemfibrozil; pregnancy or lactation; women of childbearing age not using effective methods of contraception; symptoms of muscle damage after using statins or fibrates in the past; activity of creatine kinase of more than five times the upper limit of normal.

The study is provided by the Department of Cardiology, Antoni Jurasz University Hospital No. 1 in Bydgoszcz, Poland.

After the enrollment, all participants are randomized into one of two arms receiving rosuvastatin and ezetimibe. The study drug (rosuvastatin with ezetimibe) is given: (1) in the morning $(8: 00 \mathrm{am})$ for 6 weeks and then in the evening for the next 6 weeks in the first arm; (2) in the evening $(8: 00 \mathrm{pm})$ for the first 6 weeks and then in the morning for the following 6 weeks in the second arm. In order to encourage adherence to the treatment, all patients will receive the study drug free of charge over the entire observational period. We plan to enroll 200 patients with ineffectively treated hypercholesterolemia. The scheme of the study and detailed plan of the study are presented in Figs. 1 and 2, respectively. The Standard Protocol Items: Recommendations for Interventional Trials (SPIRIT) checklist is provided as Additional file 1.

\section{Endpoints}

The primary outcome of the study is change in LDL cholesterol (LDL-C) at 6 (0 vs 6) and 12 (0 vs 12) weeks, as well as between the $6^{\text {th }}$ and 12 th weeks, of study drug treatment (combination of ezetimibe and rosuvastatin), depending on the time of day of study drug administration.

The secondary endpoints include:

- Change in total cholesterol, high-density lipoprotein cholesterol (HDL-C), triglycerides (TGs), apolipoprotein B (ApoB), apoliprotein AI (Apo AI), nonHDL-cholesterol, small, dense-LDL-cholesterol (sd-LDL-cholesterol), lipoprotein(a) at 6 (0 vs 6) and 12 (0 vs 12) weeks, as well as between the 6th and 12 th weeks of study drug treatment (combination of ezetimibe and rosuvastatin), depending on the time of day of study drug administration

- Assessment of glucose metabolism parameters: glucose, glycated hemoglobin (HbA1c) at baseline and at 6 (0 vs 6$)$ and 12 (0 vs 12) weeks, as well as between the 6 th and 12 th weeks of treatment with study drug

- Assessment of high-sensitivity C-reactive protein (hsCRP) at baseline and at 6 (0 vs 6) and 12 (0 vs 12) weeks, as well as between the 6th and 12 th weeks of treatment with study drug

- Assessment of aspartate aminotransferase (AST), alanine aminotransferase (ALT), gamma-glutamyl transferase (GGT), and creatine kinase (CK) at

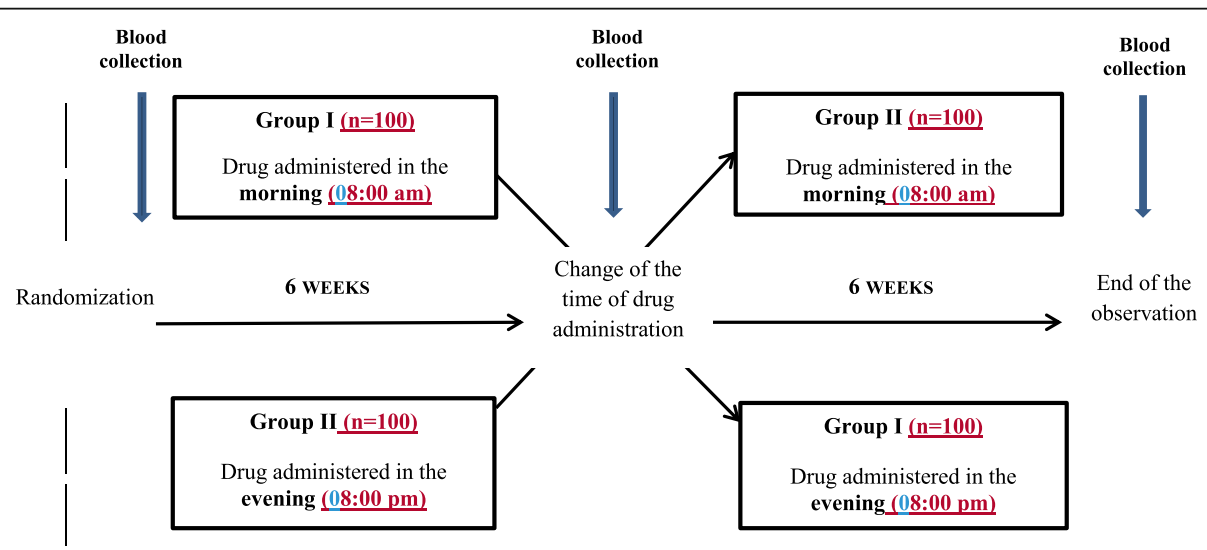

Fig. 1 Scheme of the trial 


\begin{tabular}{|c|c|c|c|c|c|}
\hline \multicolumn{6}{|c|}{ STUDY PERIOD } \\
\hline \multirow[b]{2}{*}{ TIMEPOINT } & \multirow{2}{*}{ Enrolment } & \multirow{2}{*}{$\begin{array}{c}\text { Allocation } \\
0\end{array}$} & \multicolumn{2}{|c|}{ Post-allocation } & \multirow{2}{*}{$\frac{\text { Close-out }}{t_{x}}$} \\
\hline & & & t1 ( 6 weeks) & t2 (6 weeks) & \\
\hline \multicolumn{6}{|l|}{ ENROLMENT: } \\
\hline \multirow{4}{*}{$\begin{array}{r}\text { Eligibility screen } \\
\text { Informed consent } \\
\text { Blood collection } \\
\text { Allocation }\end{array}$} & $x$ & & & & \\
\hline & $X$ & & & & \\
\hline & & $x$ & $\mathrm{X}$ & $x$ & \\
\hline & & $X$ & & & \\
\hline \multicolumn{6}{|l|}{ INTERVENTIONS: } \\
\hline \multirow{4}{*}{$\begin{array}{r}\text { Drug administered in the } \\
\text { morning [Group I] } \\
\text { Drug administered in the } \\
\text { evening [Group I] } \\
\text { Drug administered in the } \\
\text { morning [Group II] } \\
\text { Drug administered in the } \\
\text { evening [Group II] }\end{array}$} & & $x$ & $x$ & & \\
\hline & & & & $x$ & \\
\hline & & & & $\mathrm{x}$ & \\
\hline & & $x$ & $x$ & & \\
\hline \multicolumn{6}{|l|}{ ASSESSMENTS: } \\
\hline Total cholesterol & & $x$ & $\mathrm{X}$ & $x$ & \\
\hline$L D L-C$ & & $\mathrm{X}$ & $\mathrm{X}$ & $\mathrm{X}$ & \\
\hline HDL-C & & $\mathrm{X}$ & $\mathrm{X}$ & $\mathrm{X}$ & \\
\hline Triglycerides & & $X$ & $\mathrm{X}$ & $\mathrm{X}$ & \\
\hline$A p o B$ & & $\mathrm{X}$ & $X$ & $X$ & \\
\hline \multirow{3}{*}{$\begin{array}{r}\text { ApoAI } \\
\text { non-HDL-Cholesterol } \\
\text { sd-LDL-Cholesterol }\end{array}$} & & $X$ & $\mathrm{X}$ & $\mathrm{X}$ & \\
\hline & & $\mathrm{X}$ & $\mathrm{X}$ & $\mathrm{X}$ & \\
\hline & & $\mathrm{X}$ & $\mathrm{X}$ & $\mathrm{X}$ & \\
\hline lipoprotein (a) & & $X$ & $\mathrm{X}$ & $\mathrm{X}$ & \\
\hline glucose & & $\mathrm{X}$ & $\mathrm{X}$ & $\mathrm{X}$ & \\
\hline HbA1c & & $\mathrm{X}$ & $\mathrm{X}$ & $\mathrm{X}$ & \\
\hline$h s C R P$ & & $\mathrm{X}$ & $\mathrm{X}$ & $\mathrm{X}$ & \\
\hline AST & & $\mathrm{X}$ & $\mathrm{X}$ & $\mathrm{X}$ & \\
\hline$A L T$ & & $\mathrm{X}$ & $X$ & $X$ & \\
\hline CK & & $X$ & $X$ & $X$ & \\
\hline
\end{tabular}

Fig. 2 Detailed plan of the study (SPIRIT figure)

baseline and at 6 (0 vs 6) and 12 (0 vs 12) weeks, as well as between the 6th and 12th weeks of treatment with study drug

- Assessment of plasma fluorescence using stationary and time-resolved fluorescence spectroscopy at baseline, at 6 (0 vs 6$)$ and 12 (0 vs 12) weeks, as well as between the 6 th and 12 th weeks of treatment with study drug

Apart from the analysis in the whole population, the above-mentioned endpoints will be analyzed in subgroups depending on age, sex, and presence of other comorbidities.

\section{Blood sample collection and laboratory measurements} Blood collection using an intravenous catheter (VACUTAINER, Becton Dickinson, Franklin Lakes, NJ,
USA) is scheduled at the day of the enrollment and then during two follow-up visits, after 6 and 12 weeks. Laboratory tests are provided with the use of whole blood, serum, and plasma. Blood is collected in a fasting state, at least $12 \mathrm{~h}$ after the last meal, from the ulnar vein, in a volume of approximately $10 \mathrm{~mL}$. Patients are also advised to abstain from alcohol and avoid excessive physical effort within $48 \mathrm{~h}$ preceding the blood collection. Serum tubes are allowed to clot for $30 \mathrm{~min}$ in a vertical position at room temperature. Serum is separated from venous blood samples by centrifugation for $10 \mathrm{~min}$ at $3000 \times \mathrm{g}$ at room temperature. Following the centrifugation, routine laboratory measurements are performed in fresh serum (glucose, creatinine, basic lipid profile [total cholesterol, LDL-C, HDL-C, TGs], AST, ALT, GGT, CK), and only HbA1c is measured in whole blood. All remaining serum is aliquoted and stored at 
$-80{ }^{\circ} \mathrm{C}$ until assayed for hs-CRP, Apo AI, ApoB, lipoprotein(a), and sd-LDL-C. All measurements (except for HbA1c) are performed on the Horiba ABX Pentra 400 analyzer (Horiba ABX, Montpellier, France). LDL-C is measured directly and non-HDL-C is calculated. Reagents for lipoprotein(a) and sd-LDL-C (direct automated sdLDL-C kit) are supplied by Randox Laboratories (Crumlin, UK). $\mathrm{HbA1c}$ is measured on the BIO-RAD D- $10^{\mathrm{m}}$ Hemoglobin Testing System using high-performance liquid chromatography (HPLC).

Laboratory measurements are performed at the Department of Laboratory Medicine, Nicolaus Copernicus University, Collegium Medicum, Bydgoszcz, Poland, holding national and international procedures for quality control assays.

Blood samples for fluorescence measurements are collected at predefined time points. Plasma is separated from venous blood samples by centrifugation for $10 \mathrm{~min}$ at $3000 \times \mathrm{g}$ at room temperature. Plasma is filtered with the use of micro-dialyzers (Xpress Micro Dialyzer MD100, cut off $12-14 \mathrm{kDa}$ ) before fluorescence measurements. It is important to apply the preliminary fractionation to remove the majority of unnecessary particles from plasma just before the final measurement. In order to measure the fluorescence lifetime of samples, the stationary fluorescence spectrometer Hitachi F7000 and time-resolved spectrometer Life Spec II (Edinburgh Instruments Ltd) with the subnanosecond pulsed EPLED diode emitting a light of wavelength $\lambda=360 \mathrm{~nm}$ are used. The spectrometer Life Spec II is equipped with an electronically cooled photomultiplier Hamamatsu R928 connected with a TCC900 PC Card, which incorporates all the electronic modules required for time-correlated single photon counting (TCSPC). Additionally, the concentration of hydroxyproline in all samples is determined. Assessment of plasma fluorescence is provided at the Department of Pharmacology and Therapy, Nicolaus Copernicus University, Collegium Medicum.

\section{The statistical analysis}

Since there is no reference study examining the effectiveness of combined treatment of hypercholesterolemia with rosuvastatin and ezetimibe according to timing of drug administration, we decided to perform an internal pilot study of 20 patients to estimate the final sample size. The means and standard deviations of reduction in LDL-C were $53.25 \pm 31.49 \mathrm{mg} / \mathrm{dL}$ and $57.71 \pm 30.35 \mathrm{mg} / \mathrm{dL}$ during morning and evening administration, respectively. The correlation coefficient between total cholesterol reduction during morning and evening drug administration was 0.901. Based on these results and assuming a two-sided alpha value of 0.005 , we calculated using the $t$ test for dependent variables that enrollment of 157 patients would provide a $98 \%$ power to demonstrate a significant difference in total cholesterol level. To compensate for potential withdrawal of consent or loss of study participants due to other reasons, we plan to enroll 200 patients.

The statistical analysis will be carried out using the Statistica 12.0 package (StatSoft, Tulsa, OK, USA). Normal distribution of quantitative variables will be assessed with the Shapiro-Wilk test. Depending on the results of the Shapiro-Wilk test parametric, the Student $t$ tests, the one-way analysis of variance (ANOVA) or a non-parametric test (the Mann-Whitney $U$ test, Wilcoxon's signed rank test, or the Kruskal-Wallis ANOVA, multiple comparison test) will be used. The $x^{2}$ test, the $x^{2}$ with the Yates correction, or Fisher's exact test will be used for qualitative variables depending on subgroup size. To assess factors influencing plasma fluorescence parameters, correlation analysis and multiple regression analysis will be conducted. Two-sided differences will be considered significant at $p<0.05$.

\section{Safety of the trial}

The study is limited only to patients with diagnosed hypercholesterolemia, in whom statin monotherapy does not allow one to reach the therapeutic goals. Moreover, all participants receive medications of all other groups recommended by the European Society of Cardiology guidelines accordingly to their comorbidities.

\section{Discussion}

The RosEze trial is a phase IV, single-center, randomized, open-label, crossover study evaluating the effectiveness of combined therapy with rosuvastatin and ezetimibe for hypercholesterolemia depending on timing of the day of administration of the study treatment. This trial will reveal whether there is a significant difference in the effectiveness in reducing the concentration of cholesterol when the medications are taken in the morning compared with the evening time of the day. Considering that most medications are taken in the morning, it is possible that compliance with administration targets will improve if an effective dose can be taken in the morning instead of the evening.

\section{The study status}

The study is currently recruiting participants. It was registered in the ClinicalTrials.gov database with identifier NCT02772640.

\section{Additional file}

Additional file 1: SPIRIT checklist. (DOC $293 \mathrm{~kb}$ )

\section{Abbreviations}

ALT: Alanine aminotransferase; Apo Al: Apolipoprotein Al;

ApoB: Apolipoprotein B; AST: Aspartate aminotransferase; CAD: Coronary 
artery disease; CK: Creatine kinase; CVD: Cardiovascular disease; GGT: Gammaglutamyl transferase; HbA1c: Hemoglobin A1c; HDL-C: High-density lipoprotein cholesterol; Hs-CRP: High-sensitivity C-reactive protein; LDLC: Low-density lipoprotein cholesterol

\section{Acknowledgements}

We would like to thank Mrs. Teresa Skibińska for her assistance in the blood sample drawing.

\section{Funding}

The study is funded by Collegium Medicum of Nicolaus Copernicus University and did not receive any external funding (Reference number: Collegium Medicum of Nicolaus Copernicus University).

\section{Availability of data and materials}

In preparation.

\section{Authors' contributions}

$\mathrm{KO}$ conceived the study, participated in its design and its further development, and drafted the manuscript. JK participated in the design of this study and its further development and drafted the manuscript. MKa, JS, EO, and MKr participated in the design of this study and its further development. KR and NG participated in the development of the study protocol. All authors read and approved the final version of the manuscript.

\section{Ethics approval and consent to participate}

The protocol of the study was approved by the Ethics Committee of Nicolaus Copernicus University in Toruń, Ludwik Rydygier Collegium Medicum in Bydgoszcz (approval number KB 618/2015). Each participant needs to sign a written informed consent before enrollment into the study.

\section{Consent for publication}

Not applicable.

\section{Competing interests}

The authors declare that they have no competing interests.

\section{Publisher's Note}

Springer Nature remains neutral with regard to jurisdictional claims in published maps and institutional affiliations.

\section{Author details \\ ${ }^{1}$ Department of Cardiology and Internal Medicine, Nicolaus Copernicus University, Collegium Medicum, 9 Skłodowskiej-Curie Street, 85-094 Bydgoszcz, Poland. 'Department of Pharmacology and Therapy, Nicolaus Copernicus University, Collegium Medicum, 9 Skłodowskiej-Curie Street, 85-094 Bydgoszcz, Poland. '3Students Scientific Society, Department of Cardiology and Internal Medicine, Nicolaus Copernicus University, Collegium Medicum, 9 Skłodowskiej-Curie Street, 85-094 Bydgoszcz, Poland. ${ }^{4}$ Department of Laboratory Medicine, Nicolaus Copernicus University, Collegium Medicum, 9 Skłodowskiej-Curie Street, 85-094 Bydgoszcz, Poland.}

Received: 15 December 2016 Accepted: 9 June 2017

Published online: 11 July 2017

\section{References}

1. Townsend N, Nichols M, Scarborough P, Rayner M. Cardiovascular disease in Europe-epidemiological update 2015. Eur Heart J. 2015;36:2696-705.

2. Catapano AL, Graham I, De Backer G, Wiklund O, Chapman MJ, Drexel H, et al. 2016 ESC/EAS Guidelines for the Management of Dyslipidaemias: The Task Force for the Management of Dyslipidaemias of the European Society of Cardiology (ESC) and European Atherosclerosis Society (EAS) Developed with the special contribution of the European Association for Cardiovascular Prevention \& Rehabilitation (EACPR). Atherosclerosis. 2016;253:281-344.

3. Eckel RH, Jakicic JM, Ard JD, de Jesus JM, Houston Miller N, Hubbard VS, et al. 2013 AHA/ACC guideline on lifestyle management to reduce cardiovascular risk: a report of the American College of Cardiology/ American Heart Association Task Force on Practice Guidelines. Circulation. 2014;129(25 Suppl 2):S76-99.

4. Shaw K, Gennat H, O'Rourke P, Del Mar C. Exercise for overweight or obesity. Cochrane Database Syst Rev. 2006;4, CD003817.
5. Dattilo AM, Kris-Etherton PM. Effects of weight reduction on blood lipids and lipoproteins: a meta-analysis. Am J Clin Nutr. 1992;56:320-8.

6. Kelley GA, Kelley KS. Impact of progressive resistance training on lipids and lipoproteins in adults: another look at a meta-analysis using prediction intervals. Prev Med. 2009;49:473-5.

7. Huffman KM, Hawk VH, Henes ST, Ocampo Cl, Orenduff MC, Slentz CA, et al. Exercise effects on lipids in persons with varying dietary patterns - does diet matter if they exercise? Responses in Studies of a Targeted Risk Reduction Intervention through Defined Exercise I. Am Heart J. 2012;164:117-24.

8. Brown L, Rosner B, Willett WW, Sacks FM. Cholesterol-lowering effects of dietary fiber: a meta-analysis. Am J Clin Nutr. 1999;69:30-42.

9. Hollaender PL, Ross AB, Kristensen M. Whole-grain and blood lipid changes in apparently healthy adults: a systematic review and meta-analysis of randomized controlled studies. Am J Clin Nutr. 2015;102:556-72.

10. Gylling H, Plat J, Turley S, Ginsberg HN, Ellegård L, Jessup W, et al. Plant sterols and plant stanols in the management of dyslipidaemia and prevention of cardiovascular disease. Atherosclerosis. 2014;232:346-60.

11. Musa-Veloso K, Poon TH, Elliot JA, Chung C. A comparison of the LDLcholesterol lowering efficacy of plant stanols and plant sterols over a continuous dose range: results of a meta-analysis of randomized, placebocontrolled trials. Prostaglandins Leukot Essent Fatty Acids. 2011;85:9-28.

12. Yu-Poth S, Zhao G, Etherton T, Naglak M, Jonnalagadda S, Kris-Etherton PM. Effects of the National Cholesterol Education Program's Step I and Step II dietary intervention programs on cardiovascular disease risk factors: a metaanalysis. Am J Clin Nutr. 1999;69:632-46.

13. Cholesterol Treatment Trialists' (CTT) Collaboration, Baigent C, Blackwell L, Emberson J, Holland LE, Reith C, Bhala N, et al. Efficacy and safety of more intensive lowering of LDL cholesterol: a meta-analysis of data from 170000 participants in 26 randomised trials. Lancet. 2010;376:1670-81.

14. Brugts JJ, Yetgin T, Hoeks SE, Gotto AM, Shepherd J, Westendorp RG, et al. The benefits of statins in people without established cardiovascular disease but with cardiovascular risk factors: meta-analysis of randomised controlled trials. BMJ. 2009;338:b2376.

15. Mills EJ, Rachlis B, Wu P, Devereaux PJ, Arora P, Perri D. Primary prevention of cardiovascular mortality and events with statin treatments. A network metaanalysis involving more than 65,000 patients. J Am Coll Cardiol. 2008;52:1769-81.

16. Taylor F, Ward K, Moore TH, Burke M, Davey Smith G, Casas JP, et al. Statins for the primary prevention of cardiovascular disease. Cochrane Database Syst Rev. 2011;1, CD00481642.

17. Kotseva K, Wood D, De Bacquer D, De Backer G, Ryden L, Jennings C, et al. EUROASPIRE IV: a European Society of Cardiology survey on the lifestyle, risk factor and therapeutic management of coronary patients from 24 European countries. Eur J Prev Cardiol. 2016;23:636-48.

18. Agabiti Rosei E, Salvetti M. Management of hypercholesterolemia, appropriateness of therapeutic approaches and new drugs in patients with high cardiovascular risk. High Blood Press Cardiovasc Prev. 2016;23(3):217-30.

19. Valerio MG, Velayati A, Jain D, Aronow WS. Promising new therapies for the treatment of hypercholesterolemia. Expert Opin Biol Ther. 2016;16(5):609-18.

20. Holecki M, Handzlik-Orlik G, Almgren-Rachtan A, Duława J, Chudek J. The decreased achievement of therapeutic goal in lipid lowering therapy in obese and diabetic patients in Poland. Pharmacol Rep. 2017;69(1):6-12.

21. Ambegaonkar BM, Tipping D, Polis AB, Tomassini JE, Tershakovec AM. Achieving goal lipid levels with ezetimibe plus statin add-on or switch therapy compared with doubling the statin dose. A pooled analysis. Atherosclerosis. 2014;237(2):829-37.

22. Ballantyne CM, Weiss R, Moccetti T, Vogt A, Eber B, Sosef F, et al. EXPLORER Study Investigators. Efficacy and safety of rosuvastatin $40 \mathrm{mg}$ alone or in combination with ezetimibe in patients at high risk of cardiovascular disease (results from the EXPLORER study). Am J Cardiol. 2007;99:673-80.

23. Sabate E. Adherence to long-term therapies: evidence for action. Geneva: World Health Organization; 2003. http://apps.who.int/iris/bitstream/10665/ 42682/1/9241545992.pdf.

24. Benner JS, Glynn RJ, Mogun H, Neumann PJ, Weinstein MC, Avorn J. Long-term persistence in use of statin therapy in elderly patients. JAMA. 2002;288:455-61.

25. Chodick G, Shalev V, Gerber Y, Heymann AD, Silber H, Simah V, et al. Long term persistence with statin treatment in a not-for-profit health maintenance organization: a population-based retrospective cohort study in Israel. Clin Ther. 2008;30:2167-79.

26. Jackevicius CA, Mamdani M, Tu JV. Adherence with statin therapy in elderly patients with and without acute coronary syndromes. JAMA. 2002;288:462-7. 
27. McGinnis BD, Olson KL, Delate TM, Stolcpart RS. Statin adherence and mortality in patients enrolled in a secondary prevention program. Am J Manag Care. 2009;15:689-95.

28. Huser MA, Evans TS, Berger V. Medication adherence trends with statins. Adv Ther. 2005;22:163-71.

29. Kubica A, Obońska K, Fabiszak T, Kubica J. Adherence to antiplatelet treatment with $\mathrm{P} 2 \mathrm{Y} 12$ receptor inhibitors. Is there anything we can do to improve it? A systematic review of randomized trials. Curr Med Res Opin. 2016;32(8):1441-51.

30. Naderi SH, Bestwick JP, Wald DS. Adherence to drugs that prevent cardiovascular disease: meta-analysis on 376,162 patients. Am J Med. 2012 125:882-7. e1.

31. Blackburn DF, Dobson RT, Blackburn JL, Wilson TW, Stang MR, Semchuk WM. Adherence to statins, beta-blockers and angiotensin-converting enzyme inhibitors following a first cardiovascular event: a retrospective cohort study. Can J Cardiol. 2005;21:485-8.

32. Wei L, Wang J, Thompson P, Wong S, Struthers AD, MacDonald TM. Adherence to statin treatment and readmission of patients after myocardial infarction: a six year follow up study. Heart. 2002;88:229-33.

33. Corrao G, Conti V, Merlino L, Catapano AL, Mancia G. Results of a retrospective database analysis of adherence to statin therapy and risk of nonfatal ischemic heart disease in daily clinical practice in Italy. Clin Ther. 2010;32:300-10.

34. Aubert RE, Yao J, Xia F, Garavaglia SB. Is there a relationship between early statin compliance and a reduction in healthcare utilization? Am J Manag Care. 2010;16:459-66.

35. Sokol MC, McGuigan KA, Verbrugge RR, Epstein RS. Impact of medication adherence on hospitalization risk and healthcare cost. Med Care. 2005;43:521-30.

36. Mann DM, Woodward M, Muntner P, Falzon L, Kronish I. Predictors of nonadherence to statins: a systematic review and meta-analysis. Ann Pharmacother. 2010;44:1410-21.

37. Latry P, Molimard M, Dedieu B, Couffinhal T, Bégaud B, Martin-Latry K. Adherence with statins in a real-life setting is better when associated cardiovascular risk factors increase: a cohort study. BMC Cardiovasc Disord. 2011;11:46.

38. Lewey J, Shrank WH, Bowry AD, Kilabuk E, Brennan TA, Choudhry NK Gender and racial disparities in adherence to statin therapy: a meta-analysis. Am Heart J. 2013;165:665-78. e1.

39. Ryan R, Santesso N, Lowe D, Hill S, Grimshaw J, Prictor M, et al. Interventions to improve safe and effective medicines use by consumers: an overview of systematic reviews. Cochrane Database Syst Rev. 2014;4, CD007768.

40. Plakogiannis R, Cohen H. Optimal low-density lipoprotein cholesterol lowering - morning versus evening statin administration. Ann Pharmacother. 2007;41(1):106-10.

41. Martin PD, Mitchell PD, Schneck DW. Pharmacodynamic effects and pharmacokinetics of a new HMG-CoA reductase inhibitor, rosuvastatin, after morning or evening administration in healthy volunteers. Br I Clin Pharmacol. 2002;54(5):472-7.

\section{Submit your next manuscript to BioMed Central and we will help you at every step:}

- We accept pre-submission inquiries

- Our selector tool helps you to find the most relevant journal

- We provide round the clock customer support

- Convenient online submission

- Thorough peer review

- Inclusion in PubMed and all major indexing services

- Maximum visibility for your research

Submit your manuscript at www.biomedcentral.com/submit

) Biomed Central 